\title{
RISCO E GÊNERO: MEDINDO A TOLERÂNCIA AO RISCO E AS DIFERENÇAS ENTRE OS GÊNEROS
}

\author{
Alexandre Majola Gava' \\ Kelmara Mendes Vieira²
}

\section{Resumo}

Nos últimos anos, tem crescido entre os gestores de investimento e pesquisadores o interesse em conhecer o comportamento do investidor frente ao risco. A falta de um modelo padrão faz com que os profissionais financeiros desenvolvam e usem métodos simplificados geralmente limitados à simples conversações com os clientes sobre o nível de bem estar diante de diferentes cenários (GRABLE; LYTTON, 2001). Este trabalho tem dois objetivos principais. O primeiro é desenvolver e avaliar a validade de um instrumento de pesquisa para classificação dos investidores quanto à tolerância ao risco. O segundo é verificar se o gênero influência a tolerância ao risco. Foram aplicados 544 questionários em alunos de graduação e pós-graduação de sete instituições de ensino. A partir de uma Análise Fatorial, observou-se que o instrumento de pesquisa abarca três diferentes fatores de risco. As análises de correlação mostraram que, comparado à questão de risco do $\mathrm{SCF}$, o instrumento é capaz de medir outros aspectos não avaliados pela referida questão. A divisão da amostra segundo o gênero dos entrevistados mostrou que o comportamento dos participantes corrobora as pesquisas que indicam que os homens são mais propensos ao risco do que as mulheres.

Palavras-chave: Comportamento do investidor. Risco. Gênero.

\section{INTRODUÇÃO}

Nos últimos anos, tem crescido entre os gestores de investimento e pesquisadores o interesse em conhecer o comportamento do investidor frente ao risco.

\footnotetext{
' Doutor em Administração / Finanças pelo PPGA da UFRGS. Especialização na Wharton Business School (EUA). Professor na graduação da PUC-RS, CESF e FACEBG. Professor Cursos de Pós-graduação da UCS, UNESC (Universidade do Sul de Santa Catarina), PUC-RS, UNISINOS. Pontifícia Universidade Católica do Rio Grande do Sul. Endereço: Rua Eugênio Valduga, 394, Centro, Bento Gonçalves, RS, CEP95700-000. E-mail: ale.gava@terra.com.br.

2 Doutora em Administração/Finanças - PPGA/UFRGS. Professora do Curso de graduação em Administração e do mestrado em Administração da UFSM. Universidade Federal de Santa Maria. Endereço: Rua Felipe de Oliveira, I54, apto 30 I, Centro, Santa Maria, RS, CEP 970 15-250. E-mail: kelmara@smail.ufsm.br. Artigo recebido em: 23/06/2006. Aceito em 16/07/2007. Membro do Corpo Editorial Científico responsável pelo processo editorial: Prof. João Nilo Linhares
} 
A tolerância ao risco é um fator determinante na escolha sobre alocação dos ativos e, como tal, influencia diretamente na criação de produtos financeiros e na definição das estratégias de investimento.

Neste contexto, torna-se importante à distinção entre neutralidade, propensão e aversão ao risco. Dado um mesmo valor esperado, se o investidor preferir uma renda certa do que uma incerta, ele é avesso ao risco. Nesta mesma situação, se ele for indiferente entre uma renda certa e uma incerta, é neutro ao risco. Finalmente, se o investidor prefere a aposta do que a renda certa (para um mesmo valor esperado) ele é amante do risco (PINDYCK; RUBINFELD, 2004).

Os modelos de administração de investimentos requerem que os gestores de investimento utilizem, pelo menos, quatro fatores no desenvolvimento dos planos financeiros e de investimento: (1) objetivos (2) horizonte de tempo (3) estabilidade financeira e (4) tolerância ao risco (GARMAN; FORGUE, 1997 apud GRABLE; LYTTON, 1998).

Os três primeiros fatores tendem a ser objetivos e relativamente fáceis de medir. O quarto, a tolerância ao risco, refere-se a como o investidor se sente frente às subidas e quedas do mercado, com ênfase nas suas atitudes e na tolerância emocional ao risco. Ao contrário dos outros fatores, a tolerância ao risco tende a ser subjetiva e difícil de medir (GRABLE; LYTTON, 1998, HOLT; LAURY, 2002).

Embora o comportamento frente ao risco seja um elemento fundamental nas teorias sobre escolhas lotéricas, avaliação de ativos, contratos e seguros, ainda não há um consenso de como a aversão ao risco deveria ser modelada (HOLT; LAURY, 2002). A falta de um modelo padrão faz com que os profissionais financeiros desenvolvam e usem métodos "caseiros", geralmente limitados à simples conversações com os clientes sobre o nível de bem estar diante de diferentes cenários (GRABLE; LYTTON, 2001).

Do ponto de vista teórico, a teoria da utilidade continua sendo um método popular para avaliar a tolerância ao risco. Entretanto, pesquisas recentes desafiam as suposições padrões da função utilidade ao mostrar que muitos indivíduos não apresentam uma aversão ao risco constante (SHEFRIN; STATMAN, 1993). A teoria da utilidade não pode representar adequadamente as preferências e tolerâncias do tomador de risco porque as magnitudes das perdas potenciais e dos ganhos, suas chances de ocorrência e a exposição a perdas potenciais contribuem para o grau de ameaça (versus oportunidade) em uma situação de risco (KAHNEMAN; TVERSKY, 1979). Em outras palavras, pessoas tendem a serem consistentemente mais desejosas para tomar risco quando certas perdas são antecipadas e mais desejosas para mudar para ganhos seguros quando os ganhos absolutos são antecipados (STATMAN, 1995). No Brasil, a recente pesquisa de Vieira e Gava (2005) indicou que o comportamento das pessoas frente ao risco não parece seguir o esperado segundo a teoria da utilidade. 
Por outro lado, há certo consenso entre os gestores de recursos de que as variáveis demográficas podem ser usadas adequadamente para classificar os clientes em categorias de tolerância ao risco. Existem evidências de que indivíduos do sexo masculino assumem mais risco do que os do sexo feminino, conforme pode ser observado no item 2.2 deste estudo.

Este trabalho tem dois objetivos principais. O primeiro é desenvolver e avaliar a validade de um instrumento de pesquisa para classificação dos investidores quanto à tolerância ao risco. O segundo é verificar se há influência do gênero na tolerância ao risco. Para tanto é desenvolvido um questionário com dois grandes grupos de questões: um grupo para a avaliação da variável sexo e um grupo de questões para medir a tolerância ao risco.São 544 entrevistados, entre alunos de graduação e pós graduação de sete faculdades do estado do Rio Grande do Sul.

O trabalho está organizado da seguinte forma. A revisão bibliográfica (item 2) divide-se em duas seções. A primeira (item 2.1) apresenta uma discussão sobre a mensuração da tolerância ao risco. A segunda (item 2.2), é uma revisão da relação entre sexo e a tolerância ao risco. A seguir (item 3), tem-se a descrição do método de pesquisa envolvendo a definição do instrumento de pesquisa, as técnicas de análise e a amostra utilizada. A análise dos resultados envolve o procedimento de avaliação do instrumento de medida e a análise da relação entre sexo e risco (item 4). O item 5 apresenta as conclusões.

\section{REVISÃO BIBLIOGRÁFICA}

A revisão bibliográfica está dividida em duas seções. A primeira aborda os aspectos relativos à mensuração do perfil frente ao risco. A segunda é um levantamento bibliográfico da influência do gênero na tolerância ao risco.

\subsection{Tolerância ao risco}

Avaliar o nível de tolerância ao risco é um processo difícil porque tolerância ao risco é um conceito ambíguo e inconclusivo (ROZKOWSKI, 1998). Os modelos desenvolvidos nas últimas décadas podem ser classificados em cinco metodologias: escolha de dilemas, teoria da utilidade, medidas objetivas, julgamentos heurísticos e medidas subjetivas (GRABLE; LYTTON, 1999).

A escolha de dilemas era um método popular para avaliar risco até metade da década de 70. Basicamente eram desenvolvidos cenários onde os respondentes deveriam fazer uma escolha para si mesmos ou para alguém. Depois de vários anos de uso, esses testes geraram pouca evidência da propensão para aceitação do risco entre situações, principalmente porque os itens eram unidimensionais. MacCrimmon e Wehrung (1986 
Risco e gênero: medindo a tolerância ao risco e as diferenças entre os gêneros

apud GRABLE; LYTTON 1999) sumarizam os pensamentos relacionados à escolha de dilemas concluindo que itens que perguntam a alguém o "quão tolerante ao risco ele é", medem somente uma parte da natureza multidimensional do risco e que muitas pessoas cometem erros ao definir sua tolerância ao risco nessas situações.

A teoria da utilidade continua sendo um método popular para avaliar a tolerância ao risco, entretanto, sua validade vem sendo contestada principalmente devido à suposição de que a função utilidade da aversão ao risco seja constante no domínio da riqueza. A teoria da utilidade não pode representar adequadamente as preferências e tolerâncias do tomador de risco porque as magnitudes das perdas potenciais e dos ganhos, suas chances de ocorrência e a exposição a perdas potenciais contribuem para o grau de ameaça (versus oportunidade) em uma situação de risco (KAHNEMAN; TVERSKY, 1979).

Um dos contrapontos à teoria da utilidade é a teoria do prospecto. Nessa teoria:

a) as pessoas são geralmente avessas ao risco para ganhos e propensas ao risco para as perdas, sendo o segundo fator mais pronunciado;

b) pessoas atribuem pesos não lineares a ganhos e perdas potenciais;

c) resultados certos são geralmente excessivamente ponderados em comparação com resultados incertos;

d) a função utilidade é normalmente côncava para ganhos e convexa para perdas e geralmente é mais íngreme para perdas do que para ganhos;

e) pessoas fazem as escolhas sobre ganhos e perdas a partir de um ponto de referência (KAHNEMAN; TVERSKY, 1979).

Alguns autores sugerem que as medidas objetivas oferecem grande potencial para avaliar a tolerância ao risco (SCHOOLEY; WORDEN, 1996). As medidas objetivas assumem que o investidor aja racionalmente e que a alocação dos ativos é uma escolha pessoal. No entanto, medidas objetivas que requerem que o gestor deduza a tolerância ao risco de determinado indivíduo com base na sua alocação de recursos, apresenta problemas de validade. Medidas objetivas tendem a serem descritivas ao invés de preditivas, não levam em conta a natureza multidimensional do risco e muitas vezes falham na explicação do comportamento atual do indivíduo.

Os modelos heurísticos assumem que exista uma correlação forte entre características demográficas e socioeconômicas e tolerância ao risco (GRABLE; LYTTON, 1998). Por exemplo, assume-se que indivíduos velhos são menos tolerantes ao risco que indivíduos jovens. Assim, os indivíduos velhos são aconselhados a investir menos em ações e mais em títulos de renda fixa.

Alguns autores (STATMAN, 1995, GRABLE; LYTTON, 1998, entre outros) argumentam que estes métodos não são totalmente apropriados para avaliar a tolerância 
ao risco e sugerem que o melhor caminho é a utilização de medidas subjetivas. Neste caso, os autores indicam a criação de um instrumento desenhado especificamente para medir a tolerância ao risco usando cenários e situações financeiras multidimensionais. Entretanto, ainda não há medidas subjetivas ou instrumentos consolidados.

Devido à complexidade envolvida com a criação de tais medidas muitos pesquisadores têm utilizado a Survey of Consumer Finances (SCF) como uma fonte de dados para avaliar a tolerância ao risco. A Survey of Consumer Finances é uma pesquisa realizada anualmente no mercado americano e abrange dados sobre os ativos, dívidas, atitudes financeiras e comportamento financeiro de indivíduos e famílias. Nos últimos anos a pesquisa tem sido conduzida pelo National Opinion Research Center na Universidade de Chicago sob a supervisão do Federal Reserve e outras agências federais. Especificamente uma questão encontrada na SCF desde 1983 é utilizada para avaliar a tolerância a risco:

\begin{abstract}
Quais das seguintes opções é a mais próxima da quantidade de risco que você está disposto a assumir quando você economiza ou faz investimentos? 1-Assumo um risco financeiro substancial esperando ganhar um retorno substancial; 2-Assumo um risco financeiro acima da média esperando ganhar um retorno acima da média; 3-Assumo um risco financeiro médio esperando ganhar um retorno médio; 4-Não desejo assumir algum risco financeiro.
\end{abstract}

Esta é a única medida subjetiva de tolerância ao risco financeiro encontrada no SCF. O padrão de respostas tem permanecido relativamente estável ao longo dos anos, o que é um indicativo de confiabilidade. Diversas pesquisas têm utilizado a questão para avaliar a tolerância ao risco (SUNG; HANNA, 1996, GRABLE; LYTTON, 1998, DING; DEVANEY, 2000, XIAO et al., 2001, entre outros). No entanto, diversos autores levantam questões conceituais sobre a medida (GRABLE; LYTTON, 2001, HANNA et al., 2001, Xiao et al., 2001). Grable e Lytton (2001) a partir da análise de correlação testam a validade concorrente da questão SCF e argumentam que a medida pode não ser tão útil para avaliar a tolerância ao risco financeiro como previamente assumido. A partir do instrumento de risco foram definidas quatro variáveis: fator 1 , fator 2 , fator 3 e índice de risco. Cada fator é formado pela soma das respostas às questões formadoras do fator e o índice de risco é a soma das respostas atribuídas às 13 questões do instrumento. Todos os coeficientes de correlação entre a questão SCF e as quatro variáveis apresentaram resultados abaixo do limite desejado $(0,7)$, indicando que a validade concorrente da questão SCF é modesta. $\mathrm{O}$ item mede uma única dimensão da tolerância ao risco. Uma única medida tende a ser menos válida do que uma medida ampla que inclua muitos fatores, tais como a aceitação do risco no domínio das perdas ou em situações de ganhos, loterias, escolhas de investimento e decisões de alocação de recursos. 
A literatura sugere que um instrumento para avaliar a tolerância ao risco deve incluir no mínimo cinco elementos: algum conceito central de risco; permitir a derivação de uma medida de risco; relevância para os respondentes; fácil administração; e validade e confiabilidade adequadas.

Além destes elementos o instrumento deve atender a vários outros requerimentos como, por exemplo, cobrir uma variedade de situações de risco de uma maneira multidimensional, ou seja, deve incluir eventos financeiros e naturais, comportamentos, atitudes, ameaças, oportunidades e situações simples e complexas (MACCRIMMON; WEHRUNG, 1986, apud GRABLE; LYTTON, 1999).

A partir destas considerações Grable e Lytton (1999) desenvolveram um instrumento de pesquisa composto de 20 itens selecionados a partir das medidas reportadas pela literatura. Cada um dos itens, tomados individualmente, tendem a medir uma ou mais dimensões do risco. São estipuladas oito dimensões do risco, quais sejam: jogos garantidos versus prováveis; propensão para assumir risco geral; tolerância ao risco financeiro; risco como experiência e conhecimento; risco como nível de conforto; risco especulativo; teoria do prospecto; e risco do investimento.

Para explorar a multidimensionalidade do instrumento foi aplicada uma análise fatorial. Dos vinte itens descritos originalmente sete foram retirados por apresentarem cargas fatoriais inferiores a 0,45 . Os treze itens que permaneceram na análise fatorial foram distribuídos em três fatores: risco do investimento ( 5 itens), risco como conforto e experiência ( 5 itens) e risco especulativo ( 3 itens). Estes fatores englobam todas as dimensões propostas originalmente. Especificamente, os 13 itens, quando combinados trabalham juntos para avaliar a probabilidade de ganhos; a probabilidade de perdas; os potenciais ganhos em moeda; os potenciais perdas em moeda; a probabilidade mínima de sucesso dada uma ação de risco; e o retorno mínimo dada uma ação de risco. Estas situações são avaliadas pela inclusão de itens que medem escolhas e tolerâncias para jogos garantidos e prováveis, dilemas da teoria do prospecto e ganhos e perdas potenciais versus prováveis.

Para determinar a validade do instrumento os autores realizaram um teste de validade concorrente, testando o instrumento contra a medida do SCF. O coeficiente de correlação entre a questão do SCF e a o escore do instrumento foi de 0,5383 , indicando que o único item da questão do SCF não é capaz de medir as variações em todas as dimensões do instrumento.

Para aprofundar os testes de validade, Grable e Lytton (2001) aplicam o instrumento numa nova amostra e avaliam os coeficientes de correlação. São calculados coeficientes de correlação relacionando cada um dos três fatores (risco do investimento, risco como conforto e experiência e risco especulativo) do instrumento à questão do SCF. Foram obtidos, respectivamente, os seguintes coeficientes 0,51, 0,21, 0,17. Estes resultados indicam que a questão do SCF pode não ser uma medida completa da tolerância ao risco financeiro. 


\subsection{Gênero}

O trabalho de Atkinson, Baird e Frye (2003) examina a performance de fundos de investimento geridos por homens e por mulheres, concluindo que não há diferenças significativas em termos de performance, risco assumido ou outras características específicas. Há, contudo, diferenças na percepção do investidor conforme o gênero do gestor, já que, especialmente no primeiro ano, há um maior afluxo de fundos para aqueles geridos por homens. Foram examinados 144 fundos, 50\% dos mesmos geridos por homens e $50 \%$ por mulheres, a partir de regressões múltiplas.

Croson e Gneezy (2004) efetuaram uma revisão bibliográfica bastante abrangente sobre gênero e preferências por risco, afirmando que, no geral, as mulheres são mais avessas ao risco, tendo ainda menos preferência por situações competitivas do que homens. Finalmente, esta pesquisa sugere que esses fatos podem fazer com que as mulheres optem por trabalhos menos competitivos e arriscados, o que explicaria, pelo menos em parte, a diferença salarial entre sexos observada em inúmeros estudos.

No seu trabalho, Finucane et al. (2000) executaram uma pesquisa por telefone, nos EUA, atingindo 1204 pessoas maiores de 18 anos no período compreendido entre setembro de 1997 e fevereiro de 1998, obtendo uma taxa de resposta de 46,8\%. Os participantes foram convidados a indicar o nível de risco percebido (entre quase inexistente; leve; moderado ou alto) com relação a ameaças potenciais (como roubos) ou procedimentos (como transfusão de sangue). Através da comparação de médias, os resultados indicaram que homens brancos tendem a avaliar o risco como mais baixo, comparativamente a mulheres e pessoas de outras raças que não a branca.

Em seu artigo, Hudgens e Fatkin (1985) analisaram o comportamento em relação ao risco de homens e mulheres, através de um jogo computadorizado. Neste jogo, os trinta e seis participantes deveriam avaliar o risco de um campo minado, decidindo em seguida se deveriam enviar um veículo em missão através do mesmo. Os resultados, analisados a partir de testes de análise de variância (ANOVA), indicaram que homens são mais propensos ao risco do que mulheres.

Johnson e Powell (1994) examinaram o comportamento de indivíduos educados e não educados administrativamente (de maneira formal) com relação à propensão ao risco e à qualidade de suas decisões “gerenciais". A população não formalmente treinada em administração (quase seis mil casos) foi examinada a partir de seu comportamento em casas de apostas, no período de abril de 1991. Basicamente foram analisadas corridas de cavalos e de cachorros. Testes estatísticos de diferenças de médias indicaram que a qualidade das apostas foi semelhante para homens e mulheres. Por outro lado, houve sugestão de maior propensão ao risco em homens, de forma estatisticamente significativa. A população formalmente treinada consistiu de estudantes universitários de último ano em uma universidade, aos quais foi apresentado um estudo de caso que 
Risco e gênero: medindo a tolerância ao risco e as diferenças entre os gêneros

exigia a tomada de uma decisão em ambiente de risco. Foram analisados 140 estudantes, sendo que testes Qui-quadrado indicaram não haver diferenças na qualidade da decisão e tampouco na propensão ao risco entre homens e mulheres.

Masters e Meier (1988) testaram a propensão ao risco de um grupo formado por homens e mulheres, parte dos mesmos constituída de proprietários e a outra parte de gerentes de pequenos negócios. Foram analisados 50 questionários (representando uma taxa de resposta de $25 \%$ ) que solicitavam ao respondente que tomasse posição frente a decisões que envolvessem risco. A partir de testes de Qui-quadrado, afirmam os autores que não há diferenças na propensão ao risco entre proprietários de negócio e não proprietários, e tampouco entre homens e mulheres, o que, ainda segundo Masters e Meier (1988), não está de acordo com a literatura do tema.

Em resumo, a literatura indica que o nível de educação e de renda é relacionado positivamente com a tolerância para risco, enquanto que a idade relaciona-se negativamente com a mesma. Há ainda a sugestão de que o sexo masculino e a raça branca apresentam maior propensão ao risco.

\section{MÉTODO}

A descrição do método será dividida em três seções. A primeira define o instrumento de pesquisa e suas dimensões. A segunda descreve as técnicas de análise que serão utilizadas e a terceira apresenta a amostra.

\subsection{Instrumento}

Para atender aos objetivos do trabalho, foi desenvolvido um questionário com dois grandes grupos de questões (o questionário encontra-se no final do texto). $\mathrm{O}$ primeiro grupo foi construído com questões direcionadas para a avaliação da variável sexo sobre a tolerância ao risco. Foram inseridas ainda outras variáveis demográficas como variáveis de controle. (questões 1 a 9). O segundo grupo de questões tem por objetivo medir a tolerância ao risco. Foram desenvolvidas 14 questões sendo as 13 primeiras decorrentes do trabalho de Grable e Lytton (2001) (questões 10 a 22) e a última (questão 23) correspondendo à questão do SCF.

As treze questões referentes à tolerância ao risco buscam avaliar as diferentes dimensões do risco, conforme assinalado no Quadro 1.

A validade do questionário enquanto instrumento de pesquisa, segundo Kahneman e Tversky (1979), é reforçada pelo fato de que, neste tipo de metodologia, é possível o teste de um grande número de questões teóricas. Além disso, parte-se do pressuposto de que as pessoas sabem como agiriam numa situação real de escolha e respondem ao questionário com esta predisposição, não tendo ainda nenhum incentivo para esconder suas reais preferências. 


\begin{tabular}{|c|c|c|c|c|c|c|c|}
\hline 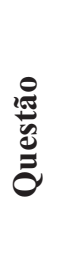 & 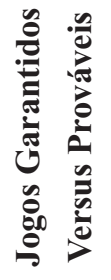 & 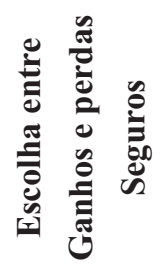 & 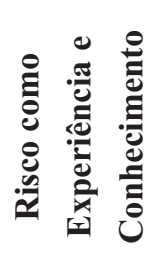 & 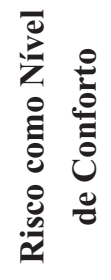 & 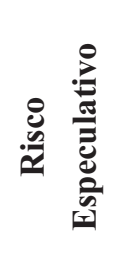 & 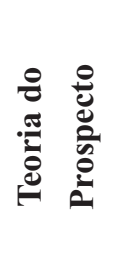 & 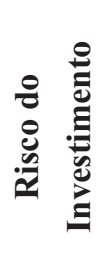 \\
\hline 10 & & & $\mathrm{X}$ & X & & & \\
\hline 11 & $X$ & & & & $X$ & & \\
\hline 12 & & & & $X$ & & & \\
\hline 13 & & & $X$ & & & & $X$ \\
\hline 14 & & & $X$ & X & & & X \\
\hline 15 & & & $\mathrm{X}$ & $X$ & & & \\
\hline 16 & & & $\mathrm{X}$ & & $X$ & & $X$ \\
\hline 17 & $X$ & $X$ & & & $\mathrm{X}$ & & \\
\hline 18 & & & & & & $X$ & \\
\hline 19 & & & & & & $\mathrm{X}$ & \\
\hline 20 & & & $\mathrm{X}$ & & & & $\mathrm{X}$ \\
\hline 21 & & & & $\mathrm{X}$ & & $\mathrm{X}$ & \\
\hline 22 & $X$ & & & & $\mathrm{X}$ & & \\
\hline
\end{tabular}

Quadro 1: Dimensões do Risco Abordadas por Cada Questão

Fonte: adaptado de Grable e Lytton (1999)

\subsection{Técnicas de Análise}

Para uma análise expliratória do instrumento de pesquisa optou-se pela utilização da Análise Fatorial.A Análise Fatorial é um modelo de mensuração das relações entre os indicadores (variáveis observadas) e os fatores. Conforme Hair et al. (1999), a análise fatorial aborda o problema de como analisar a estrutura de inter-relações (covariâncias) existentes entre um grande número de variáveis com a definição de uma série de dimensões subjacentes comuns, conhecidas como fatores.

Para verificar se a análise fatorial é adequada para um determinado conjunto de dados sugere-se a aplicação de dois testes. A fim de avaliar se a correlação existente entre as variáveis é significativa, a ponto de apenas alguns fatores serem capazes de representar grande parte da variabilidade dos dados aplica-se o teste de esfericidade de Bartlett. Além disso, deve-se verificar se a correlação entre cada par de variáveis pode ser explicada pelas demais variáveis incluídas no estudo. Então, calcula-se a medida de Kaiser-Meyer-Olkin (KMO) onde valores iguais ou menores do que 0,5 indicam que a análise fatorial é insatisfatória para a explicação da correlação de cada par de variáveis pelas demais variáveis consideradas no estudo. 
Risco e gênero: medindo a tolerância ao risco e as diferenças entre os gêneros

Existem diversos métodos para a estimação das cargas fatoriais, mas dois se destacam: o método das componentes principais e o método da máxima verossimilhança. Neste trabalho, optou-se pelo método das componentes principais para a estimação das cargas fatoriais, pois segundo Malhotra (2001), este método é mais recomendado quando a preocupação principal do pesquisador é determinar o número mínimo de fatores, que respondem pela variância máxima nos dados para utilização em análises multivariadas subseqüentes.

É possível encontrar tantos fatores, quantas forem as variáveis pesquisadas. Entretanto, geralmente o pesquisador busca resumir as informações contidas nas variáveis originais em um número menor de fatores. Para isso, é preciso definir entre os métodos disponíveis qual será o critério de determinação do número de fatores a serem utilizados. Hair et al. (1999) apresenta diferentes métodos para escolha do número de fatores: determinação a priori; autovalores; gráfico de declive; percentagem da variância; confiabilidade meio a meio e testes de significância. Neste trabalho, optou-se pelo método dos autovalores superiores a um, pois deseja-se que cada fator explique a variância de pelo menos uma variável.

Entretanto, nem sempre os fatores escolhidos são de fácil interpretação e às vezes, recorre-se à rotação dos eixos. Neste trabalho optou-se pelo método varimax normalizado, por ser um método ortogonal que simplifica os fatores pela obtenção de cargas fatoriais altas em algumas variáveis e baixas em outras.

Após a Análise Fatorial, foi utilizada a Análise de Correlação para comparar o instrumento desenvolvido para a avaliação da tolerância ao risco à questão do SCF.

O coeficiente de Correlação de Spearman é a versão não-paramétrica do coeficiente de correlação de Pearson. O coeficiente assume valores entre - 1 e 1 . O sinal do coeficiente indica a direção da relação e o seu valor absoluto à força da relação, com valores altos indicando forte relação.

Posteriormente, para a avaliação da influência da variável gênero para a determinação da tolerância ao risco foram adotados dois procedimentos. Inicialmente foi aplicado o teste de Qui-quadrado e o teste t para avaliar se existe diferença entre os sexos, para as demais variáveis de controle. Posteriormente, os testes Qui-quadrado e $t$ foram novamente utilizados para avaliar a relação entre sexo e as medidas de risco. O teste Qui-quadrado foi aplicado para as variáveis com escala nominal ou ordinal e o teste t para as variáveis de razão.

\subsection{Amostra}

Obteve-se uma amostra de alunos de graduação e pós graduação em Administração de sete instituições diferentes, localizadas nas cidades de Santa Maria, Caxias do Sul, Porto Alegre, São Leopoldo e Farroupilha. Foram entrevistados alunos matriculados em disciplinas da área financeira (Matemática Financeira, Administração 
Financeira, Tópicos Especiais em Finanças, Gestão de Risco). Tal critério de seleção teve como objetivo garantir que os entrevistados já tinham alguma noção de risco e de produtos financeiros. Dentre os alunos matriculados nestas disciplinas foram entrevistados todos aqueles que compareceram às aulas entre os dias 03 e 07 de abril de 2006. A Tabela 1 apresenta o percentual de questionários em cada instituição.

Tabela 01: Número e Percentual de Questionários Aplicados por Instituição

\begin{tabular}{l|c|c}
\hline \multicolumn{1}{c|}{ Instituição } & Número de Questionários & Percentual \\
\hline CESF & 86 & 15,81 \\
UCS & 35 & 6,43 \\
PUC -RS & 77 & 14,15 \\
Faculdade Palotina & 52 & 9,56 \\
UNISINOS & 151 & 27,76 \\
UNIFRA & 87 & 15,99 \\
ULBRA & 56 & 10,29 \\
\hline TOTAL & $\mathbf{5 4 4}$ & $\mathbf{1 0 0 , 0 0}$ \\
\hline
\end{tabular}

Fonte: dados da pesquisa

\section{ANÁLISE DOS RESULTADOS}

As Tabelas de Freqüência foram construídas a partir do número de entrevistados que responderam à questão, ou seja, os valores percentuais ignoram os não respondentes e determinam os percentuais a partir do número de respostas obtidas. Dos entrevistados, $49,1 \%$ são do sexo masculino e $50,9 \%$ do sexo feminino. A grande maioria é solteira ( $72,4 \%$ solteiros e $25 \%$ casados $)$, não tem filhos $(83,1 \%)$, está cursando a graduação $(86,2 \%)$ e, em relação à raça, se considera uma pessoa branca $(92,1 \%)$. Já a renda média dos 442 entrevistados que declararam renda é de R \$1.160,00. A idade média é 26 anos. As Tabelas 2 e 3 descrevem o perfil dos entrevistados quanto à ocupação e moradia.

Tabela 2: Freqüência e Percentual dos Entrevistados Segundo a Ocupação.

\begin{tabular}{c|c|c}
\hline Ocupação & Frequência & Percentual \\
\hline Neg. Próprio Formal & 61 & 11,21 \\
Neg. Próprio Informal & 12 & 2,21 \\
Funcionário Público & 28 & 5,15 \\
Funcionário E. Privada & 284 & 52,21 \\
Funcionário ONG & 5 & 0,92 \\
Não Trabalha & 104 & 19,12 \\
Outra & 50 & 9,19 \\
\hline Total & $\mathbf{5 4 4}$ & $\mathbf{1 0 0 , 0 0}$ \\
\hline
\end{tabular}

Fonte: dados da pesquisa 
Risco e gênero: medindo a tolerância ao risco e as diferenças entre os gêneros

Tabela 3: Freqüência e Percentual dos Entrevistados Segundo a Moradia.

\begin{tabular}{c|c|c}
\hline SCF & Frequência & Percentual \\
\hline Risco Substancial & 128 & 23,79 \\
Risco Acima da Média & 96 & 17,84 \\
Risco Médio & 196 & 36,43 \\
Sem Risco & 118 & 21,93 \\
\hline Total & $\mathbf{5 3 8}$ & $\mathbf{1 0 0 , 0 0}$ \\
\hline
\end{tabular}

Fonte: dados da pesquisa

Mais da metade dos entrevistados trabalha em empresa privada. A maioria ainda mora com os pais, o que é de certa forma era esperado, já que a maioria ainda é jovem e solteira.

A freqüência de respostas à questão de risco do SCF, dá uma idéia geral da posição dos entrevistados frente ao risco (Tabela 4).

Tabela 4: Freqüência e Percentual dos Entrevistados Segundo a Questão do SCF.

\begin{tabular}{c|c|c}
\hline Fatores & $\begin{array}{c}\text { Variância Explicada } \\
\text { \% }\end{array}$ & $\begin{array}{c}\text { Variância Acumulada } \\
\text { \% }\end{array}$ \\
\hline 1 & 25,719 & 25,719 \\
2 & 14,220 & 39,939 \\
3 & 13,299 & 53,238 \\
\hline
\end{tabular}

Fonte: dados da pesquisa

$\mathrm{Na}$ amostra de Grable e Lytton (2001) a maioria dos entrevistados era do sexo masculino (55\%), casados (72\%), brancos (90\%), com idade média de 43 anos e com renda entre $\$ 20.000,00$ e $\$ 90.000,00$. Os percentuais obtidos para a questão do SCF foram, respectivamente, $4 \%, 22 \%, 56 \%$ e $18 \%$.

Comparando os resultados das duas pesquisas observa-se que nesta os entrevistados assumiram mais risco substancial e relativamente menos risco médio do que os entrevistados de Grable e Lytton. Tais resultados seriam esperados já que os entrevistados desta pesquisa são mais jovens e solteiros, características que sugerem uma maior propensão ao risco.

Para a Análise Fatorial foram utilizadas as questões de 10 a 22 do questionário. As questões de 01 a 09 não foram utilizadas na análise pois são questões de controle. Optou-se por adotar a análise de componentes principais como método de extração dos fatores e, como método de rotação, aplicou-se à rotação varimax normalizada. Como critério de extração foi definido autovalor maior que um.

As questões 11, 12, 18, 19, e 22 apresentaram índices de comunalidade abaixo do nível de comunalidade aceitável e foram retiradas do estudo. Para avaliar o efeito da retirada de tais variáveis, o modelo também foi estimado com as mesmas. Como 
a variância explicada não é alterada significativamente, manteve-se a decisão de retirá-las. Os testes de adequação e especificidade da amostra de dados foram satisfatórios. $\mathrm{O} \mathrm{KMO}$ apresentou um coeficiente de 0,681 . O teste de especificidade de Bartlett assumiu o valor 308,57 significativo a 1\%. A Tabela 5 apresenta o percentual de variância explicada por cada fator.

Tabela 5: Percentual de Variância Explicada por Cada Fator.

\begin{tabular}{c|c|c}
\hline Moradia & Frequência & Percentual \\
\hline Moro com os Pais & 303 & 55,80 \\
Residência Própria & 145 & 26,70 \\
Residência Alugada & 79 & 14,50 \\
Outro & 16 & 3,00 \\
\hline Total & $\mathbf{5 4 3}$ & $\mathbf{1 0 0 , 0 0}$ \\
\hline
\end{tabular}

Fonte: dados da pesquisa

Observe que o primeiro fator sozinho explica 25,7\% da variância. Em conjunto os três fatores explicam $53 \%$ de toda a variância. A matriz rotada apresenta quais as variáveis mais importantes dentro de cada um dos fatores.

Tabela 6: Cargas Fatoriais dos Três Fatores Obtidos com a Rotação Varimax.

\begin{tabular}{c|c|c|c}
\hline \multirow{2}{*}{ Questões } & \multicolumn{3}{|c}{ Fatores } \\
\cline { 2 - 4 } & $\mathbf{1}$ & $\mathbf{2}$ & $\mathbf{3}$ \\
\hline 13 & $\mathbf{0 , 8 2 6}$ & 0,026 & 0,042 \\
14 & $\mathbf{0 , 7 2 2}$ & 0,114 & $-0,033$ \\
10 & 0,014 & $\mathbf{0 , 7 6 9}$ & 0,069 \\
15 & 0,149 & $\mathbf{0 , 7 0 1}$ & $-0,046$ \\
21 & 0,403 & $\mathbf{0 , 4 0 5}$ & 0,301 \\
16 & $-0,061$ & $-0,172$ & $\mathbf{0 , 7 6 2}$ \\
17 & $-0,056$ & 0,345 & $\mathbf{0 , 5 5 9}$ \\
20 & 0,379 & 0,081 & $\mathbf{0 , 5 5 7}$ \\
\hline
\end{tabular}

Fonte: dados da pesquisa

Observa-se que dois dos três fatores apresentam três questões relevantes. Apenas o fator 1 é formado por apenas duas questões com coeficientes altos. Todos os coeficientes ficam acima do valor 0,30, considerado o mínimo desejável para amostras superiores a 350 casos (HAIR et al., 1999).

Uma análise mais detalhada das variáveis que compõem cada fator permite uma avaliação da dimensão do risco preponderante em cada fator. As questões 13, 14 têm em comum a dimensão do risco do investimento e a dimensão do risco como experiência e conhecimento. O fator 2, com as variáveis 10,15 e 21, aborda preponderantemente o 
aspecto de quão confortável o indivíduo se sente frente a situações de risco. As questões 16, 17 e 20, do fator 3, abordam preponderantemente aspectos do risco especulativo.

A partir dos resultados obtidos pela Análise Fatorial foram construídos três fatores e um índice de risco. Cada fator corresponde à soma das variáveis preponderantes para o mesmo. Por exemplo, o fator 1 foi construído a partir das respostas dadas às questões 13 e 14. O índice de risco é a soma das respostas atribuídas às 8 questões.

Com o objetivo de avaliar a relação entre o instrumento de medida de risco e a questão sobre risco do SCF, foram calculados os coeficientes de correlação de Spearman. A questão SCF foi avaliada tanto na escala nominal quanto na escala ordinal. A escala ordinal, se refere à questão como apresentada originalmente aos entrevistados, ou seja, com quatro respostas possíveis. A questão SCF- Nominal foi criada com apenas duas categorias, sendo atribuído o valor 1 para o entrevistado que assume algum nível de risco (risco substancial, risco acima da média, risco médio) e o valor zero para o entrevistado que não assume risco. Os resultados são apresentados na Tabela 7.

Tabela 7: Coeficientes de Correlação de Spearman.

\begin{tabular}{l|c|c}
\hline \multicolumn{1}{c|}{ Variáveis } & Coeficiente de Correlação & Significância \\
\hline SCF-Nominal x Fator 1 & 0,130 & 0,003 \\
SCF-Nominal x Fator 2 & 0,187 & 0,000 \\
SCF-Nominal x Fator 3 & 0,114 & 0,009 \\
SCF-Nominal x Risco Financeiro & 0,186 & 0,000 \\
SCF-Ordinal x Fator 1 & 0,211 & 0,000 \\
SCF-Ordinal x Fator 2 & 0,283 & 0,000 \\
SCF-Ordinal x Fator 3 & 0,203 & 0,000 \\
SCF-Ordinal x Risco Financeiro & 0,318 & 0,000 \\
\hline
\end{tabular}

Fonte: dados da pesquisa

Todos os coeficientes são significativos e positivos, mas baixos indicando que a questão do SCF sozinha não é capaz de abarcar todas as dimensões do risco financeiro abrangidas no instrumento de pesquisa.

Antes de avaliar a relação entre a propensão ao risco e o sexo do entrevistado, buscou-se identificar se existe homogeneidade no que se refere as variáveis de controle para os dois grupos formados segundo o sexo do entrevistado. Para tanto, dividiuse a amostra em dois grupos (masculino e feminino) e para as variáveis nominais ou ordinais, foi aplicado o teste Qui-Quadrado e para as variáveis demográficas de razão utilizou-se o teste t.

A alta concentração de respostas em uma única classe exigiu que as variáveis ocupação, raça e grau de instrução fossem re-classificadas para permitir um agrupamento das classes com baixo número de casos. A variável ocupação passou a ser formada por quatro classes: Negócio Próprio, Funcionário de Empresa, Não Trabalha 
e Outro. A maioria dos respondentes que assinalou a opção outro especificou que exerciam a função de estagiário. A variável raça passou a ter apenas duas classes: Branca e Outra. A variável grau de instrução também foi dividida em duas classes: Graduação Incompleto e Outro.

Os resultados demonstraram que não há relação entre sexo e as variáveis estado civil, raça e instrução. $O$ teste qui-quadrado foi significativo apenas para a relação entre sexo e ocupação. O resultado é apresentado na Tabela 8.

Tabela 8: Valor de Qui-quadrado e Significância para a Relação entre as Variáveis Ocupação e Sexo.

\begin{tabular}{l|c|c|c|c|c|c|c}
\hline \multirow{2}{*}{\multicolumn{1}{c|}{ Sexo }} & \multicolumn{4}{|c|}{ Ocupação } & \multicolumn{2}{c}{ Qui-quadrado } \\
\cline { 2 - 8 } & Négocio Próprio & Funcionário & Não Trabalha & Outra & Total & valor & sig \\
\hline Masculino & 50 & 145 & 50 & 20 & 265 & & \\
Feminino & 22 & 170 & 53 & 30 & 275 & 14,78 & 0,002 \\
Total & 72 & 315 & 103 & 50 & 540 & & \\
\hline
\end{tabular}

Fonte: dados da pesquisa

Portanto, para a amostra selecionada não há diferenças entre os entrevistados do sexo masculino e do sexo feminino quanto à Estado Civil, Raça e Grau de Instrução. Já para a variável Ocupação (Tabela 08) observa-se que há uma diferença significativa entre os sexos.

Para as variáveis de razão, aplicou-se o teste $t$ de diferença de médias. Para determinar se o teste $t$ deveria ser homocedástico ou heterocedástico, previamente foi aplicado um teste F para igualdade de variâncias. Nos casos em que a hipótese de igualdade das variâncias foi aceita (rejeitada) aplicou-se o teste $t$ homocedástico (heterocedástico). Os resultados são apresentados na Tabela 9.

Tabela 9: Valores de t e Significância para a Diferença de Médias entre os Sexos, Segundo as variáveis Idade, Número de Filhos, Filhos Dependentes e Renda.

\begin{tabular}{|c|c|c|c|c|c|c|}
\hline \multirow{2}{*}{ Variável } & \multirow{2}{*}{ Sexo } & \multirow{2}{*}{ Casos } & \multirow{2}{*}{ Média } & \multirow{2}{*}{ Desvio Padrão } & \multicolumn{2}{|c|}{ Teste $t$} \\
\hline & & & & & valor & sig \\
\hline \multirow{2}{*}{ Idade } & Masculino & 259 & 26,47 & 7,63 & \multirow{2}{*}{2,630} & \multirow{2}{*}{0,009} \\
\hline & Feminino & 272 & 24,85 & 6,51 & & \\
\hline \multirow{2}{*}{$N^{o}$ Filhos } & Masculino & 265 & 0,31 & 0,7 & \multirow{2}{*}{1,942} & \multirow{2}{*}{0,053} \\
\hline & Feminino & 275 & 0,21 & 0,56 & & \\
\hline \multirow{2}{*}{ Filhos Dependentes } & Masculino & 262 & 0,29 & 0,68 & \multirow{2}{*}{1,619} & \multirow{2}{*}{0,106} \\
\hline & Feminino & 273 & 0,21 & 0,58 & & \\
\hline \multirow{2}{*}{ Renda } & Masculino & 216 & 1378 & 1567 & \multirow{2}{*}{3,567} & \multirow{2}{*}{0,000} \\
\hline & Feminino & 223 & 951 & 810 & & \\
\hline
\end{tabular}

Fonte: dados da pesquisa 
Com relação ao número de filhos e aos filhos dependentes, em média, não há diferença entre os sexos. Por outro lado, os homens têm idade ligeiramente superior à das mulheres e percebem uma renda média maior. De certa maneira estes resultados seriam esperados já que historicamente, os homens recebem mais que as mulheres para uma mesma ocupação e, ainda, como mais mulheres estão desempregadas ou exercem outra profissão (maioria estagiárias) era esperado que a renda média das mulheres fosse inferior à dos homens.

De maneira geral, pode-se concluir que a amostra é relativamente homogênea no que se refere às variáveis de controle. Tal homogeneidade é desejável para que se possa amenizar a interferência do efeito destas variáveis no estudo da relação entre o sexo do indivíduo e sua tolerância ao risco.

Os resultados para a diferença entre sexo e a medida de risco do SCF são apresentados nas Tabelas 10 e 11.

Tabela 10: Valor de Qui-quadrado e Significância para a Relação entre as Variáveis SCF Nominal e Sexo.

\begin{tabular}{c|c|c|c|c|c}
\hline \multirow{2}{*}{ SCF Nominal } & \multicolumn{3}{|c|}{ Sexo } & \multicolumn{2}{c}{ Qui-quadrado } \\
\cline { 2 - 6 } & Masculino & Feminino & Total & valor & sig \\
\hline Não Assume Risco & 39 & 223 & 262 & & \\
Assume Risco & 77 & 195 & 272 & 14,14 & 0,000 \\
Total & 116 & 418 & 534 & & \\
\hline
\end{tabular}

Fonte: dados da pesquisa

Tabela 11: Valor de Qui-quadrado e Significância para a Relação entre as Variáveis SCF Ordinal e Sexo.

\begin{tabular}{c|c|c|c|c|c}
\hline \multirow{2}{*}{ SCF Ordinal } & \multicolumn{3}{|c|}{ Sexo } & \multicolumn{2}{c}{ Qui-quadrado } \\
\cline { 2 - 6 } & Masculino & Feminino & Total & valor & sig \\
\hline Risco Substancial & 59 & 68 & 127 & & \\
Risco Acima da Média & 66 & 30 & 96 & & \\
Risco Médio & 98 & 97 & 195 & 26,41 & 0,000 \\
Sem Risco & 39 & 77 & 116 & & \\
Total & 262 & 272 & 534 & & \\
\hline
\end{tabular}

Fonte: dados da pesquisa

Tanto para a medida ordinal como para a medida nominal a diferença entre os sexos é significativa. Avaliando a Tabela 10 observa-se que o percentual de mulheres que não assume risco (53\%) é bem superior ao de homens (34\%). Já a Tabela 11 , mostra que os homens são mais propensos a assumir riscos acima da média do que as mulheres. Os resultados dos testes de diferença de média entre os sexos para os fatores de risco (Tabela 12) confirmam essa tendência. 
Tabela 12: Valores de T e Significância para a Diferença de Médias entre os Sexos, Segundo os Fatores 1, 2 e 3 e Risco Financeiro

\begin{tabular}{|c|c|c|c|c|c|c|}
\hline \multirow{2}{*}{ Variável } & \multirow{2}{*}{ Sexo } & \multirow{2}{*}{ Casos } & \multirow{2}{*}{ Média } & \multirow{2}{*}{ Desvio Padrão } & \multicolumn{2}{|c|}{ Teste $\mathbf{t}$} \\
\hline & & & & & valor & sig \\
\hline \multirow{2}{*}{ Fator 1} & Masculino & 264 & 3,67 & 1,13 & \multirow{2}{*}{3,657} & \multirow{2}{*}{0,000} \\
\hline & Feminino & 271 & 3,33 & 1,03 & & \\
\hline \multirow{2}{*}{ Fator 2} & Masculino & 260 & 6,44 & 1,41 & \multirow{2}{*}{2,522} & \multirow{2}{*}{0,012} \\
\hline & Feminino & 268 & 6,13 & 1,36 & & \\
\hline \multirow{2}{*}{ Fator 3} & Masculino & 254 & 6,58 & 1,85 & \multirow{2}{*}{2,013} & \multirow{2}{*}{0,045} \\
\hline & Feminino & 270 & 6,26 & 1,83 & & \\
\hline \multirow{2}{*}{ Risco Financeiro } & Masculino & 252 & 16,68 & 3,07 & \multirow{2}{*}{3,549} & \multirow{2}{*}{0,000} \\
\hline & Feminino & 264 & 15,73 & 3,02 & & \\
\hline
\end{tabular}

Fonte: dados da pesquisa

Observa-se que a variável sexo apresenta valores de $\mathrm{t}$ significativos para as quatro variáveis utilizadas para medir risco, indicando que, em média, há diferença entre os sexos na assunção de risco. Observe ainda que a média do grupo masculino é superior à média do feminino para todas as variáveis, confirmando que os homens são mais propensos ao risco.

Cabe finalmente ressaltar que Weber e Hsee (1998) afirmam que pode haver diferenças na percepção do risco que sejam determinantes na atitude do indivíduo frente ao risco. Segundo este enfoque, o comportamento frente ao risco poderia ser determinado a partir de diferenças no risco percebido. Este tipo de trabalho tem encontrado diferenças significativas na percepção de risco quando testando culturas diferentes, tais como no trabalho de Bontempo, Bottom e Weber (1997), segundo o qual indivíduos de Taiwan e Hong Kong apresentam diferenças de percepção para risco com relação a indivíduos dos EUA e Países Baixos. Contudo, este mesmo estudo não encontra diferenças significativas em função de outros fatores, tais como renda ou tipo de ocupação, o que sinaliza que os resultados encontrados no presente estudo não são devidos a diferenças de percepção entre os respondentes, dada a sua homogeneidade cultural.

\section{CONCLUSÕES}

Profissionais do mercado financeiro sejam atuando como pesquisadores, consultores, administradores ou analistas, compartilham do objetivo comum de avaliar, tão rapidamente quanto possível, as tolerâncias e preferências dos indivíduos quanto ao risco financeiro. Em muitos casos, não se tem mais do que uma descrição qualitativa e julgamentos subjetivos para se avaliar o efeito das crenças, sentimentos, necessidades e aspirações dos indivíduos sobre a sua tolerância ao risco. No entanto, 
avaliar o nível de tolerância ao risco é um processo difícil porque tolerância ao risco é um conceito ambíguo e inconclusivo (ROZKOWSKI, 1998).

Nas últimas décadas, os modelos desenvolvidos para análise de risco têm sido classificados em diversas metodologias. Dentre elas os modelos heurísticos, que assumem que exista uma correlação forte entre características demográficas e socioeconômicas e tolerância ao risco. Entretanto, alguns autores defendem a utilização de medidas subjetivas e recomendam a criação de um instrumento multidimensional. Assim, este trabalho buscou desenvolver e avaliar um instrumento que pudesse medir a tolerância ao risco e ao mesmo tempo avaliar o efeito do sexo, um dos aspectos heurísticos.

Das 13 questões desenvolvidas para medir a tolerância ao risco, 08 se mostraram aptas a permanecer na Análise Fatorial. Após a rotação foram obtidos três fatores com autovalores superiores a um. As questões 13, 14 têm em comum a dimensão do risco do investimento e a dimensão do risco como experiência e conhecimento. $\mathrm{O}$ fator 2, com as variáveis 10, 15 e 21, aborda preponderantemente o aspecto de quão confortável o indivíduo se sente frente a situações de risco.As questões 16, 17 e 20, do fator 3, abordam preponderantemente aspectos do risco especulativo.

A partir dos resultados obtidos para a Análise Fatorial foram construídos três fatores e um índice de risco financeiro. Com o objetivo de avaliar a relação entre o instrumento de medida de risco e a questão sobre risco do SCF, foram calculados os coeficientes de correlação de Spearman. Os resultados mostram uma correlação positiva, mas baixa, indicando que a questão do SCF sozinha não é capaz de abarcar todas as dimensões do risco financeiro abrangidas no instrumento de pesquisa.

Previamente à avaliação da relação entre a tolerância ao risco e a variável sexo buscou-se identificar a relação entre a variável sexo e as demais variáveis de controle (raça, idade, instrução, ocupação, filhos, estado civil). A amostra foi dividida em dois grupos (masculino e feminino) e para as variáveis nominais ou ordinais, foi aplicado o teste Qui-Quadrado e para as variáveis de razão utilizou-se o teste t. Os testes mostraram que, de maneira geral, a amostra é relativamente homogênea no que se refere às variáveis de controle. Tal homogeneidade era desejável para que se pudesse amenizar a interferência do efeito destas variáveis no estudo da relação entre o sexo do indivíduo e sua tolerância ao risco.

Para avaliar a relação entre sexo e tolerância ao risco, aplicou-se novamente o teste qui quadrado, para as questões do SCF, e o teste $t$, para os fatores de risco. A significância dos testes confirmou as evidências empíricas de que os homens são mais tolerantes ao risco do que as mulheres.

Este artigo apresentou uma dentre as diversas maneiras possíveis para avaliação da tolerância ao risco. Obviamente mais pesquisas são necessárias para confirmar os resultados. O estudo poderá ser replicado em amostras diferentes ou mesmo ser comparado a outros instrumentos ou questões de avaliação da tolerância ao risco para verificação da confiabilidade e validade do instrumento. 


\title{
RISK AND GENDER: MEASURING RISK TOLERANCE AND GENDER DIFFERENCES
}

\begin{abstract}
In recent years there has been a growing interest among researchers and fund managers in understanding investor's behavior towards risk. The lack of a standard model leads financial professionals to develop and use simplified methods, usually limited to simple conversations with clients about their level of well being in a variety of scenarios (GRABLE; LYTTON, 2001). The present article has two main objectives. The first objective is to develop and to verify the validity of a research instrument for the classification of investors according to their risk tolerance. The second objective is to verify if gender has an influence on risk tolerance. A total of 544 questionnaires were applied to undergraduate and post-graduate students of seven Brazilian universities. A Factor Analysis established that there are three different risk factors in this research. When compared to the SCF question, the correlation analysis showed that the research questionnaire is able to determine other factors. The segmentation of the sample according to gender showed that women are more risk-averse than men, as documented by other studies.
\end{abstract}

Keywords: Investor behavior. Risk. Gender.

\section{REFERÊNCIAS}

ATKINSON, S. M.; BAIRD, S. B.; FRYE, M. B. Do female mutual fund managers manage differently? Journal of Financial Research, v. 26, n. 1, p. 1-18, 2003.

BONTENPO, R. N.; BOTTOM, W. P.; WEBER, E. U. Cross-cultural differences in risk perception: A model-based approach. Risk Analysis, v. 17, p. 479-488, 1997.

CROSON, R.; GNEEZY, U. Gender differences in preferences. Working paper, University of Pennsylvania, 2005.

DING, J.; DEVANEY, S.A. Predictors of emergency funds adequacy. Proceedings of Association for Financial Counseling and Planning Education, p.56-65, 2000.

FINUCANE, M. L. et al. Gender, race, and perceived risk: the 'white male' effect. Health, Risk \& Society, v. 2, n. 2, 2000. 
Risco e gênero: medindo a tolerância ao risco e as diferenças entre os gêneros

GARMAN, E. T.; FORGUE, R. E. Personal Finance, 5.ed, Boston:Houghton Mifflin, 1997.

GRABLE, J.; LYTTON, R.H. Investor risk tolerance: testing the efficacy of demographics as differentiating and classifying factors. Financial Counseling and Planning, v.9, p.61-73, 1998;

. Financial risk tolerance revisited: the development of a risk assessment instrument. Financial Services Review, v.8, p.163-181, 1999.

Assessing the concurrent validity of the SCF risk tolerance question. Financial Counseling and Planning, v.12, p.43-52, 2001.

HAIR, Joseph F. et al. Análisis multivariante. 5. ed., Madrid: Prentice-Hall, 1999.

HANNA, S. D.; GUTTER, M.; FAN, J. A measure risk tolerance based on economic theory. Financial Counseling and Planning, v.12, p. 53-60, 2001

HOLT, C.A.; LAURY, S.K. Risk aversion and incentive effects. The American Economic Review, v. 92, n. 5, p.1644-1655, 2002.

HSEE, C. K.; WEBER, E. U. Cross-cultural differences in risk perception, but Crosscultural similarities in attitudes towards perceived risk. Management Science, v. 44, n. 9, 1998.

HUDGENS, G. A., FATKIN, L. Sex differences in risk taking: repeated sessions on a computer simulated task. The Journal of Psychology, v. 119, n. 3, p.197-206, 1985.

JOHNSON, J. E. V., POWELL, P. L. Decision making, risk and gender: are managers different? British Journal of Management, n. 5, p. 123-138, 1994.

KAHNEMAN, D.; TVERSKY, A. Prospect theory: an analysis of decision under risk. Econometrica, v. 47, p. 263-291, 1979.

MACCRIMMON, K.R.; WHRUNG, D.A. Risk management. New York: The Free Press, 1986.

MALHOTRA, N. K. Pesquisa de marketing: uma orientação aplicada. 3. ed. Porto Alegre: Bookman, 2001. 
MASTERS, R.; MEIER, R. Sex differences and risk taking propensity of entrepreneurs. Journal of Small Business Management, p. 31-35, jan. 1988.

PINDYCK, R.; RUBINFELD, D. Microeconomics. New York, Prentice Hall, 2004.

ROSKOWSKI, M. J. Risk tolerance in financial decisions. In: Readings in Financing Planning, Bryn Mawr, PA: The American Colege, p. 281-328, 1998.

SCHOOLEY, D.K.; WORDEN, D.D. Risk aversion measures: comparing attitudes and asset allocation. Financial Services Review, v. 5, p. 87-99, 1996.

SHEFRIN, H., STATMAN, M. Behavioral aspects of the design and marketing of financial products. Financial Management, v. 22, n. 2, p.123-134, 1993.

STATMAN, M. A behavioral framework for dollar-cost averaging. The Journal of Portfolio Management, p. 70-78, 1995.

SUNG, J.; HANNA, S. Factors related to risk tolerance. Financial Conseling and Planing, v. 8, p. 47-58, 1996.

VIEIRA, K. M., GAVA, A. M. Tomada de Decisão em Ambiente de Risco: uma Avaliação sob a Ótica Comportamental. In: ENCONTRO NACIONAL DA ASSOCIAÇÃ̃O NACIONAL DE PROGRAMAS DE PÓS-GRADUAÇÃO EM ADMINISTRAÇÃO. ENANPAD, 29, 2005, Brasília. Anais... 2005.

XIAO, J. J. et al. Attitude toward risk and risk-taking behavior of business-owning families. The Journal of Consumer Affairs, v. 35, n. 2, p. 307, 2001.

\section{QUESTIONÁRIO}

Prezado(a) Entrevistado(a):

Este questionário faz parte de uma pesquisa científica que tem por objetivo avaliar as preferências dos indivíduos frente às diversas situações que envolvem a tomada de decisão em ambiente de risco. Sua participação é vital para a consecução do projeto. Por favor, responda as questões abaixo, sabendo que não existem respostas "corretas", mas apenas alternativas diferenciadas segundo a percepção do respondente. Agradecemos a sua participação! 
Risco e gênero: medindo a tolerância ao risco e as diferenças entre os gêneros

1. Sexo: 1.1 ( ) Masculino 1.2 ( ) Feminino

2. Idade: anos

3. Estado Civil:

3.1 ( ) Casada(o)

3.2 ( ) Solteira(o)

3.3 ( ) Separada(o)

3.4 ( ) Viúva (o)

4. Tem filhos?

4.1 ( ) Não.

4.2 ( ) Sim: Quantos filhos?

Quantos ainda são dependentes financeiramente?

5. Ocupação:

5.1 ( ) Negócio Próprio Formal

5.2 ( ) Negócio Próprio Informal

5.3 ( ) Funcionário Público

5.4 ( ) Funcionário de Empresa Privada

5.5 ( ) Funcionário de Organização Não Governamental (ONG)

5.6 ( ) Não trabalha

5.7 ( ) Outro. Especificar:

6. Seu Salário/Renda Média Mensal: R\$

7.Em relação à raça, você se considera uma pessoa:

7.1 ( ) indígena

7.2 ( ) branca

7.3 ( ) preta

7.4 ( ) amarela

7.5 ( ) parda

8. Qual o seu grau de instrução?:
8.1 ( ) Primeiro Grau Incompleto
8.2 ( ) Primeiro Grau Completo
8.3 ( ) Ensino Médio Incompleto
8.4 ( ) Ensino Médio Completo
8.5 ( ) Graduação Incompleto
8.6 ( ) Graduação Concluída
8.7 ( ) Curso Técnico
8.8 ( ) Especialização 
8.9 ( ) Mestrado

8.10 ( ) Doutorado

9. Com relação à moradia:

9.1 ( ) Moro com os pais

9.2 ( ) Possuo residência própria

9.3 ( ) Moro em residência alugada

9.4 ( ) Outro. Especificar:

10. Em geral, como o seu melhor amigo o descreveria como um tomador de risco?

10.1 ( ) Um jogador

10.2 ( ) Alguém desejoso de assumir riscos após uma pesquisa adequada

10.3 ( ) Um cauteloso

10.4 ( ) Uma pessoa avessa a risco

11. Você está participando de um jogo na TV e pode escolher entre as opções seguintes. O que você escolheria?

11.1 ( ) $\mathrm{R} \$ 1.000,00$ em dinheiro

11.2 ( ) $50 \%$ de chance de ganhar R $\$ 5.000,00$

11.3 ( ) $25 \%$ de chance de ganhar R $\$ 10.000,00$

11.4 ( ) 5\% de chance de ganhar R\$ $100.000,00$

12. Você acabou de economizar para as "férias dos seus sonhos", mas, três semanas antes da viagem, é demitido do seu trabalho. Você:..

12.1 ( ) Cancela as férias

12.2 ( ) Escolhe uma viagem muito mais modesta

12.3 ( ) Faz o programado, porque você precisa de tempo para se preparar para buscar um novo trabalho

12.4 ( ) Amplia suas férias, porque esta pode ser sua última chance de viajar na primeira classe

13. Se você inesperadamente recebesse $R \$ 20.000,00$ para investir, o que você faria?

13.1 ( ) Depositaria na conta corrente bancária

13.2 ( ) Investiria em um título de dívida de alta qualidade ou num fundo de renda fixa 13.3 ( ) Investiria em ações ou em fundos de ações

14. Em termos de experiência, como você se sentiria investindo em ações ou fundos de ações? 
Risco e gênero: medindo a tolerância ao risco e as diferenças entre os gêneros

14.1 ( ) Desconfortável

14.2 ( ) Confortável

14.3 ( ) Muito confortável

15. Quando você pensa na palavra "risco" qual das palavras seguintes vem a sua mente primeiro?

15.1 ( ) Perda

15.2 ( ) Incerteza

15.3 ( ) Oportunidade

15.4 ( ) Emoção

16. Alguns especialistas estão prevendo que o preço de ativos como ouro, jóias, coleções e imóveis (ativos reais) devem aumentar de valor e que os preços dos títulos de dívida devem cair. Entretanto, os especialistas tendem a concordar que os títulos públicos de dívida são relativamente seguros. A maioria dos seus investimentos está aplicada em títulos públicos de dívida com alta taxa de juros. O que você faria?

16.1 ( ) Manteria os títulos

16.2 ( ) Venderia os títulos e aplicaria metade do dinheiro no mercado financeiro e a outra metade em ativos reais

16.3 ( ) Venderia os títulos e aplicaria todo o dinheiro em ativos reais

16.4 ( ) Venderia os títulos, aplicaria todo o dinheiro em ativos reais e ainda tomaria emprestado recursos adicionais para comprar mais ativos reais

17. Dados os retornos possíveis oferecidos nos quatro investimentos abaixo, qual deles você prefere?

17.1 ( ) $\mathrm{R} \$ 200,00$ de ganho no melhor resultado; R\$ 0 no pior resultado

17.2 ( ) $\mathrm{R} \$ 800,00$ de ganho no melhor resultado; $\mathrm{R} \$ 200,00$ de perda no pior resultado

17.3 ( ) $\mathrm{R} \$ 2.600,00$ de ganho no melhor resultado; $\mathrm{R} \$ 800,00$ de perda no pior resultado

17.4 ( ) $\mathrm{R} \$ 4.800,00$ de ganho no melhor resultado; $\mathrm{R} \$ 2.400,00$ de perda no pior resultado

18. Imagine que você acabou de ganhar R $\$ 1.000,00$. Agora você deve escolher entre:

18.1 ( ) um ganho certo de $\mathrm{R} \$ 500,00$

18.2 ( ) 50\% de chance de ganhar $\mathrm{R} \$ 1.000,00$ e $50 \%$ de chance de não ganhar nada

19. Agora imagine que você acabou de ganhar R\$2.000,00. Então, você deve escolher entre: 
19.1 ( ) uma perda certa de $\mathrm{R} \$ 500,00$

19.2 ( ) 50\% de chance de perder $\mathrm{R} \$ 1.000,00$ e $50 \%$ de chance de não perder nada

20.Supondo-se que um parente tivesse deixado-lhe uma herança de $\mathrm{R} \$ 100.000,00$, determinando ainda que todo o dinheiro deveria ser investido em apenas uma das seguintes opções, qual delas você escolheria?

20.1 ( ) Poupança ou fundo de renda fixa

20.2 ( ) Um fundo que possui ações e títulos

20.3 ( ) Um portfólio de 15 ações

20.4 ( ) Ativos como ouro, prata e petróleo

21. Se você tem $\mathrm{R} \$ 20.000,00$ para investir, qual das seguintes opções de investimento você acharia mais atrativa?

21.1 ( ) 60\% em investimentos de baixo risco; $30 \%$ em investimentos de risco médio e $10 \%$ em investimentos de alto risco

21.2 ( ) 30\% em investimentos de baixo risco; $40 \%$ em investimentos de risco médio e $30 \%$ em investimentos de alto risco

21.3 ( ) 10\% em investimentos de baixo risco; $40 \%$ em investimentos de risco médio e $50 \%$ em investimentos de alto risco

22. Seu vizinho e amigo de confiança, um experiente geólogo, está formando um grupo para investir na exploração de uma mina de ouro. O investimento de risco poderia pagar entre 50 e 100 vezes o valor investido, caso seja um sucesso. Seu amigo estima que a chance de sucesso é de somente $20 \%$. Em caso de fracasso, todo valor investido seria perdido. Se você tivesse o dinheiro, quanto investiria?

22.1 ( ) Nada

22.2 ( ) Um mês de salário

22.3 ( ) Três meses de salário

22.4 ( ) Seis meses de salário

23. Qual das afirmações seguintes é a mais próxima da quantidade de risco que você está disposto a assumir quando você economiza ou faz investimentos?

23.1 ( ) Assumo um risco financeiro substancial esperando ganhar um retorno substancial

23.2 ( ) Assumo um risco financeiro acima da média esperando ganhar um retorno acima da média

23.3 ( ) Assumo um risco financeiro médio esperando ganhar um retorno médio

23.4 ( ) Não desejo assumir algum risco financeiro 